Annuaire du Collège de France 2017-2018

\title{
Galaxies et cosmologie
}

\section{Françoise Combes}

\section{OpenEdition}

Journals

Édition électronique

URL : https://journals.openedition.org/annuaire-cdf/15501

DOI : 10.4000/annuaire-cdf.15501

ISBN : 978-2-7226-0572-5

ISSN : 2109-9227

Éditeur

Collège de France

Édition imprimée

Date de publication : 30 décembre 2020

Pagination : 69-81

ISBN : 978-2-7226-0516-9

ISSN : 0069-5580

Référence électronique

Françoise Combes, "Galaxies et cosmologie », L'annuaire du Collège de France [En ligne], 118 | 2020,

mis en ligne le 01 avril 2021, consulté le 22 août 2022. URL : http://journals.openedition.org/annuairecdf/15501 ; DOl : https://doi.org/10.4000/annuaire-cdf.15501 


\title{
GALAXIES ET COSMOLOGIE
}

\author{
Françoise COMBES
}

Membre de l'Institut (Académie des sciences), professeure au Collège de France

Mots-clés : galaxies, barres, spirales, trous noirs, interaction de marée, étoiles, absorption, quasars

La série de cours et séminaires "Dynamique des galaxies, spirales et barres, interactions et fusions " est disponible, en audio/et ou en vidéo, sur le site internet du Collège de France (https://www.college-de-france.fr/site/francoise-combes/course-2017-2018.htm).

\section{ENSEIGNEMENT}

COURS ET SÉMINAIRES - DYNAMIQUE DES GALAXIES, SPIRALES ET BARRES, INTERACTIONS ET FUSIONS

\section{Introduction}

La formation et l'évolution des galaxies dépendent essentiellement de leur dynamique, de leur gravité et de l'hydrodynamique du gaz. Ces phénomènes contrôlent la formation d'étoiles, et tous les processus associés, y compris les rétroactions dues à l'énergie des supernovae. Chaque galaxie croît en symbiose avec son trou noir supermassif, tapi en son centre, qui devient actif par accrétion de gaz de façon cyclique, avec des périodes typiques de 40 millions d'années.

Les premières galaxies à se former sont très riches en gaz. Par dissipation, elles forment très vite un disque en rotation. Puis progressivement, les petites galaxies interagissent et fusionnent pour former des galaxies plus grosses. Le gaz est consommé pour former des étoiles, et la fusion de systèmes stellaires n'est plus dissipatif. Les disques sont alors détruits, et le moment angulaire s'annule et se dilue, pour former des systèmes sphéroïdaux, avec peu ou pas de rotation.

Les galaxies à disque possédant du gaz sont la plupart du temps spirales. Les ondes spirales ou barrées sont nécessaires pour avoir des forces tangentielles et des 
couples, qui peuvent échanger le moment angulaire, et permettre à la galaxie d'accréter de la matière, de se concentrer et de former ses étoiles. Les barres et les spirales sont le véritable moteur de l'évolution.

Les grands surveys de galaxies, et notamment le Sloan Digital Sky Survey (SDSS), ont révélé une bimodalité caractéristique des galaxies : dans un diagramme couleurmagnitude, les galaxies se distribuent en une séquence rouge de galaxies plus massives en fin d'évolution, et en un nuage bleu de galaxies qui forment encore des étoiles et qui sont moins massives. Les processus qui peuvent expliquer l'arrêt brutal de la formation d'étoiles dans les galaxies rouges sont encore mal connus. La fraction de ces galaxies rouges croît avec la masse et l'environnement. Il pourrait s'agir de phénomène de balayage de gaz dû à la pression dynamique, les interactions de marée, ou alors la stabilisation du gaz à cause de la croissance des bulbes. Les phénomènes de rétroaction, soit des noyaux actifs, soit des supernovae, apparaissent réversibles.

\section{Cours 1 - Dynamique stellaire : orbite et résonances}

\section{Séminaire - Morphologie des galaxies}

Valérie de Lapparent (IAP, Paris), le 20 novembre 2017

Dans ce premier cours, nous soulignons, par un bref rappel historique, que ce n'est qu' au $\mathrm{XX}^{\mathrm{e}}$ siècle que les astronomes ont pris conscience de l'existence des galaxies, ces mondes à part comme la nôtre, la Voie lactée. Auparavant, les galaxies apparaissaient à leurs yeux comme des nébuleuses, raison pour laquelle la galaxie d'Andromède a par exemple porté le nom de "nébuleuse d'Andromède ». Vers 1920, lors d'un débat entre Harlow Shapley et Heber Curtis, le premier soutient que les nébuleuses font partie de notre Galaxie, de même que les amas globulaires. La taille de la Voie lactée lui semble très grande $(100 \mathrm{kpc})$, et les étoiles dans les amas globulaires lui apparaissent géantes. Henrietta Leavitt a pourtant découvert en 1909 la relation période-luminosité des céphéides, et montré qu'il s'agit d'indicateurs de distance très intéressants. Vesto Slipher a également mesuré les vitesses des nébuleuses par effet Doppler : elles sont majoritairement en récession, mais cela doit être dû à la pression de radiation. Heber Curtis pense quant à lui que la taille de la Voie lactée est plutôt de l'ordre de 10kpc, et que les nébuleuses lointaines sont des galaxies extérieures à la nôtre. Edwin Hubble identifie des céphéides dans M31 et M33 : ce sont des galaxies composées aussi de milliards d'étoiles, et non pas de gaz. Elles sont distantes de $\sim 1000 \mathrm{kpc}$. Il interprète les vitesses positives comme une expansion de l'Univers en 1929.

La dynamique des galaxies est établie sur la gravité et l'hydrodynamique du gaz. Les étoiles constituent un milieu sans collision, et leur densité dans l'espace des phases est conservée. On peut définir des critères de stabilité (critère de Toomre), et déterminer quelles sont les ondes de densité spirales qui vont pouvoir se développer dans les disques, selon leur dispersion de vitesses. Des régions critiques sont celles des résonances de Lindblad, lorsqu'il existe un rapport rationnel entre période de rotation et oscillation épicyclique. Les amas globulaires sont des systèmes stellaires internes aux galaxies et ne possèdent pas de matière noire, de par leur formation. Ces amas sont des systèmes plus compacts que les galaxies à masse comparable, bien qu'il existe un endroit de continuité entre galaxies naines compactes et amas globulaires. 


\section{Cours 2 - Théorie des ondes de densité \\ Séminaire - Évolution temporelle des vitesses des barres et spirales dans les galaxies}

Daniel Pfenniger (Observatoire de Genève), le 27 novembre 2017

Les bras spiraux d'une galaxie ne sont pas seulement des caractéristiques esthétiques, mais sont le moyen qu'a une galaxie de faire croître sa masse et d'accréter du gaz. En effet, de la matière tend à s'effondrer sur les galaxies en permanence à partir des filaments cosmiques, mais celle-ci doit d'abord se débarrasser de son moment angulaire, avant de pouvoir tomber vers le centre et y former de nouvelles étoiles. Pour ce faire, il faut des structures non axisymétriques, comme les barres et les spirales, qui sont à l'origine de forces tangentielles et de couples qui peuvent transférer le moment angulaire vers l'extérieur. C'est sur ce principe qu'est fondée la théorie des ondes de densité de Lin \& Shu. Les bras spiraux ne sont pas des bras matériels qui s'enroulent et se diluent rapidement par rotation différentielle, mais des ondes de densité qui voyagent à vitesse de rotation constante. Pourtant, ces ondes ne sont pas éternelles, et s'amortissent très vite, surtout en présence de gaz et de chocs. Il faut alors envisager des mécanismes de génération d'ondes et de maintenance de ces ondes. L'amplification du swing montre comment un paquet d'ondes leading s'amplifie considérablement en se transformant en trailing, lorsque la fréquence épicyclique et la self-gravité se conjuguent à la rotation différentielle. On peut alors imaginer des cycles de wasers, analogue aux masers (ou lasers), où des ondes sont amplifiées à la corotation, et réfléchies au centre, à la résonance de Lindblad interne. La présence d'une barre d'étoiles, ou l'interaction entre galaxies, produisent les mécanismes nécessaires pour engendrer la formation de la première onde spirale. Toutefois, les ondes de densité cohérentes sur tout le disque sont rares, et l'on observe plutôt des morceaux qui se régénèrent avec le mécanisme du swing. Les simulations cosmologiques récentes, qui contiennent une quantité de matière noire beaucoup plus importante qu'observée en réalité, tendent à stabiliser les disques, et voient de moins en moins d'ondes de densité spirales.

\section{Cours 3 - Instabilités et amplification des ondes}

\section{Séminaire - Instabilités et formation stellaire : la diversité dans l'universalité}

Florent Renaud (Université de Surrey, Angleterre), le 4 décembre 2017

La génération des ondes de densité spirales fait encore couler beaucoup d'encre. D'autres mécanismes pourraient être considérés en dehors des ondes courantes, amplifiées par le swing. Par exemple, les ondes pourraient être des modes propres. De plus, de nombreuses ondes pourraient se développer dans les disques, interagissant entre elles de façon non linéaire, comme par exemple des barres primaire et nucléaire, ou des ensembles couplés barres-spirales. Les ondes pourraient ainsi se développer jusqu'au centre, et même sortir dans les zones externes des galaxies, là où règne le gaz atomique HI. Des harmoniques peuvent se développer, comme des instabilités en forme d'éperons, de plumes, de branchements. Le champ magnétique pourrait intervenir, et générer des instabilités de Parker, qui produisent des paquets réguliers, à l'origine de la formation d'étoiles comme des perles sur un 
collier. Les spirales et les barres stellaires produisent des ondes de choc dans le composant gazeux. Ceci peut provoquer la condensation du gaz en étoiles et peut expliquer la brillance des bras spiraux par rapport aux interbras, alors que le contraste de densité n'est pas énorme.

Comment diagnostiquer la présence d'ondes de densité spirales ? S'il existe un fort contraste dans le potentiel gravitationnel, on devrait voir des perturbations de vitesse caractéristiques à la traversée des bars. Ces diagnostics sont parfois observés et parfois non, peut-être à cause de perturbations locales (explosion de supernovae, interactions, etc.).

Les simulations numériques aident à comprendre les phénomènes, par exemple la formation de disques épais, les phénomènes de feedback des supernovae, le flaring, etc.

\section{Cours 4 - Formation et évolution des barres}

\section{Séminaire - Barres et structures morphologiques : traceurs de la formation et moteurs de l'évolution des galaxies}

\section{Frédéric Bournaud (CEA-Saclay), le 11 décembre 2017}

La majorité des galaxies spirales (les deux tiers) sont barrées. Les barres sont l'un des moteurs pour la formation d'ondes de densité spirales cohérentes, avec les interactions de galaxies. Le comportement des galaxies barrées peut se comprendre en considérant les familles d'orbites des étoiles, qui se concentrent autour des orbites périodiques. Au centre des galaxies, les orbites sont parallèles à la barre, ce sont les orbites x1. Puis, à chaque résonance, ces orbites tournent de $90^{\circ}$, et sont donc perpendiculaires à la barre à l'extérieur de la corotation. Comme la barre n'est pas soutenue par les orbites stellaires, elle s'arrête à la corotation, comme confirmé par les simulations numériques. Ce phénomène permet d'estimer la vitesse de rotation des ondes barrées, qui ne peut pas se mesurer directement. S'il existe deux résonances internes de Lindblad (ILR), les orbites stellaires seront alors perpendiculaires à la barre entre les deux ILR. Il s'agit d'un phénomène d'autorégulation des barres : avec le temps la barre piège de plus en plus d'étoiles, et s'étend en rayon. Sa vitesse moyenne décroît, elle ralentit, car la vitesse de précession des orbites décroît avec le rayon. Une barre plus lente commence à créer une ILR, puis deux. Ainsi la barre va elle-même stopper sa croissance, en permettant à des orbites de s'orienter dans la direction perpendiculaire. La barre primaire ainsi fragilisée va voir une barre secondaire, plus petite et plus rapide, se découpler au centre. Souvent, les deux barres s'échangent de l'énergie, en interagissant de façon non linéaire à une résonance commune. Cela peut être la corotation de la barre nucléaire, qui correspond à l'ILR de la barre primaire.

Les simulations numériques ont accru notre connaissance des phénomènes de barres et spirales dans les galaxies à disque : nous avons notamment appris que les résonances de Lindblad se produisent aussi dans la direction perpendiculaire au plan. Il se forme alors comme une cacahuète, qui forme un pseudo-bulbe de galaxies, très reconnaissable dans les galaxies barrées vues par la tranche. Il se forme aussi des anneaux de formation stellaire aux résonances, et les barres sont un moyen d'amener le gaz au centre et d'alimenter les trous noirs. 


\title{
Cours 5 - Séquence rouge et nuage bleu ou séquence de Hubble
}

\section{Séminaire - Séquence rouge et nuage bleu : une enquête sur l'origine de la bimodalité des galaxies}

\author{
David Elbaz (CEA-Saclay), le 18 décembre 2017
}

La formation d'étoiles dans les galaxies est assez mystérieuse, et se produit en divers épisodes. Dans les grands surveys de galaxies, on s'aperçoit qu'il existe une bimodalité entre les galaxies rouges, qui ne forment plus d'étoiles, i.e. qui sont mortes, et les galaxies bleues, encore très actives, que l'on appelle « séquence principale ». Cette distinction correspond grossièrement à la classification de Hubble en galaxies elliptiques (ou early-type) rouges, et galaxies à disques, ou spirales, bleues, de type tardif (late-type). Comment la formation d'étoiles est-elle stoppée brutalement, afin d'expliquer deux séquences bien séparées, avec une transition uniquement peuplée de peu de galaxies, appelée «vallée verte»? Lorsque l'on remonte le temps, c'est-à-dire lorsqu' on observe loin dans l'Univers, on s'aperçoit que les galaxies formaient beaucoup plus d'étoiles qu'aujourd'hui, au moins vingt fois plus. Mais il y avait aussi une séquence bleue, la séquence principale, et déjà des galaxies elliptiques mortes, la séquence rouge.

Il a été observé que la fraction de galaxies rouges croît nettement en fonction de la masse et de l'environnement. Plusieurs mécanismes ont été avancés pour expliquer la chute brutale de formation d'étoiles dans les galaxies. Il est possible de couper lentement l'approvisionnement en gaz froid à partir des filaments cosmiques. Dans les halos très massifs, le gaz en tombant est chauffé dans des chocs, et ne peut plus former d'étoiles. L'effet de l'environnement d'amas de galaxies peut plus rapidement étouffer les galaxies, et les balayer de leur gaz par pression dynamique, ou forces de marée. D'autre part, la formation des bulbes de galaxies par interaction et fusion va stabiliser le gaz: c'est une stabilisation par la morphologie. Les phénomènes de feedback (rétroaction) des supernovae et des noyaux actifs peuvent aussi éjecter le gaz brutalement, mais, en général, cela n'est pas définitif, car le gaz revient dans la galaxie. Les observations ont en outre montré que les effets de rétroaction pouvaient même être positifs, c'est-à-dire que l'activité du noyau actif pouvait engendrer une formation d'étoiles.

\section{Cours 6 - Galaxies elliptiques : dynamique et plan fondamental}

\section{Séminaire - Galaxies de type précoce : dynamique et processus de formation}

Eric Emsellem (ESO, Garching, Allemagne), le 8 janvier 2018

Les galaxies elliptiques, et plus généralement early-type, sont des galaxies sphéroïdales, dont la dynamique stellaire est fondée sur la dispersion de vitesses. Parfois, elles sont aplaties, mais ce n'est pas par rotation comme pour les spirales. Des études détaillées de leur cinématique ont montré dans les années 1980 que les galaxies elliptiques ne tournent pas ou très peu. Ces galaxies sont formées par fusions de galaxies spirales, ce qui peu à peu annule le moment angulaire. Les premières galaxies étant très gazeuses, forment d'abord un disque par dissipation du gaz. Puis la fraction de gaz diminue considérablement, et la fusion de systèmes purement stellaires conduit à des sphéroïdes, soutenus par une dispersion de vitesse 
anisotrope. La dernière direction de fusion domine et détermine la direction de plus grande élongation de la galaxie finale.

Bien sûr le scénario plus réaliste n'est pas aussi simple, car une galaxie elliptique de champ peut continuer à accréter du gaz des filaments cosmiques, et reformer un disque. Les études récentes de ces galaxies rouges ont montré qu'elles possèdent toutes un disque, même d'importance faible, et qu'elles ont souvent un résidu de rotation. On distingue les rotateurs rapides et les rotateurs lents, qui eux sont le résultat de plusieurs fusions.

La forme à 3D des galaxies sphéroïdales peut être soit oblate (les deux axes égaux sont les grands axes), ou prolate (les deux axes égaux sont les petits axes). Parfois la forme est celle d'un triaxe. La preuve de la fusion de galaxies à l'origine des elliptiques se trouve dans l'existence de coquille d'étoiles autour, soit dans un plan équatorial (cas oblate), soit alignées sur le grand axe d'une galaxie prolate. Dans ce cas, les coquilles sont alternées, les plus jeunes se formant au centre. Le nombre de coquilles permet de dater l'événement de fusion, et la distribution radiale des coquilles donne des renseignements sur la distribution de matière noire, moyennant la connaissance de la friction dynamique passée.

Les relations d'échelle des galaxies elliptiques sont plus complexes que pour les spirales : outre la relation de Faber-Jackson, analogue à la relation de Tully-Fisher, on peut définir un plan fondamental.

\section{Cours 7 - Friction dynamique et formation hiérarchique}

\section{Séminaire - La dynamique de l'Univers : des grandes structures aux petits groupes de galaxies}

\section{Gary Mamon (IAP, Paris), le 15 janvier 2018}

Comment se forment les galaxies ? L'une des premières hypothèses consistait à imaginer un effondrement brutal ou progressif d'un seul système sur lui-même, qui formerait rapidement des étoiles, en résultant dans une galaxie elliptique, ou bien un halo d'étoiles vieilles et un disque de gaz et d'étoiles jeunes, selon la vitesse relative de l'effondrement et de la formation d'étoiles. Un autre scénario, le scénario hiérarchique, qui est privilégié aujourd'hui, consiste à penser que les premières galaxies sont toutes des disques (à cause du gaz), et que la fusion de galaxies annule le moment angulaire et détruit les disques, pour former des galaxies early-type. Il faut rajouter à celui-ci le fait que la plupart des baryons sont encore en dehors des galaxies, donc une grande partie de la matière est accrétée à partir des filaments cosmiques, encore aujourd'hui.

Pour quantifier le scénario hiérarchique, il faut pouvoir estimer exactement la friction dynamique, qui fait fusionner les galaxies ensemble. Le processus est délicat et chaotique, surtout la friction à distance. Et la formule de Chandrasekhar, très utilisée pour avoir une estimation analytique, n'est souvent pas valable. Il faut essayer d'estimer la friction avec des simulations numériques, mais celles-ci sont en général loin de la réalité, à cause des résolutions, spatiales et en masse, limitées.

Les calculs de friction permettent d'estimer la vitesse de formation des bulbes par fusions, la vitesse des barres dans les halos de matière noire, la durée de vie des groupes compacts de galaxies, selon la distribution de matière noire, etc. On voit que la principale friction est due à la matière noire, et c'est pourtant le composant le plus inconnu. Cela permettra peut-être de contraindre les modèles de matière noire. En ce 
moment, le modèle standard de matière noire froide $(\mathrm{CDM})$ parvient difficilement à expliquer la grande fréquence de galaxies sans bulbe autour de nous.

\section{Cours 8 - Interactions entre galaxies}

\section{Séminaire - Interactions et fusions de galaxies : à la recherche du passé de la Voie lactée}

Paola di Matteo (Obs-Paris, Meudon), le 22 janvier 2018

Les galaxies autour de nous sont fréquemment en interaction : bras de marée, pont entre galaxies. Les galaxies sont nées en groupe et sous-structures, et interagissent davantage que si leurs positions étaient aléatoires dansle ciel. Le catalogue des 338 galaxies particulières (en interaction) conçu par Halton Arp est même paru en 1966, juste après l'Atlas des galaxies normales en 1961. Jusqu'en 1972, les astronomes pensaient que ces interactions étaient magnétiques, car la matière était comme canalisée dans des tubes de force. Mais les simulations rudimentaires des frères Toomre en 1972 ont montré que des interactions de marée, appliquées aux ensembles peu liés des galaxies, pouvaient former des ponts et des bras spiraux aussi fins. Les forces de marée à distance peuvent en effet se décomposer en deux termes de Fourier, soit le terme d'ordre $2, \cos (2$ theta), dans le plan du disque des galaxies, et le terme d'ordre 1, cos(theta), perpendiculaire au plan. Ainsi deux bras spiraux vont s'enrouler dans le plan, qui lui va être gauchi comme une crêpe.

Les simulations numériques ont montré comment les interactions de marée pouvaient reproduire de façon remarquable les morphologies des galaxies en interaction. Selon le paramètre d'impact, les bras spiraux peuvent se refermer en anneau, et ainsi les collisions frontales peuvent expliquer les galaxies an roue de charrette, comme la Cartwheel. Dans le Groupe local, les Nuages de Magellan sont en interaction entre eux, et avec la Voie lactée. Ils vont bientôt fusionner avec notre Galaxie. De même, Andromède est en interaction avec la Voie lactée, et les deux spirales les plus massives du Groupe local vont sans doute fusionner dans 2 à 3 milliards d'années pour former une galaxie plus rouge, ou elliptique. Plus généralement, on peut expliquer la formation d'anneaux polaires autour des galaxies, la formation des coquilles autours des galaxies elliptiques, la formation des flambées de formation d'étoiles lors de la fusion de spirales riches en gaz, etc. Finalement, l'histoire de la formation d'étoiles, qui possède un pic vers $\mathrm{z}=2$, peut s'expliquer par le plus grand taux d'interaction et de fusion entre galaxies, quand les amas se virialisent.

\section{ENSEIGNEMENT À L'EXTÉRIEUR - LES TROUS NOIRS SUPERMASSIFS ET LEUR RÔLE COSMOLOGIQUE}

Cours à la FRAMA (Fédération de recherche André-Marie Ampère) de l'université Claude Bernard Lyon 1, les 14 et 21 mars

\section{Introduction}

Il est bien établi aujourd'hui que toutes les galaxies à bulbe possèdent un trou noir supermassif en leur centre, de un million à plusieurs milliards de masses solaires. Le rapport de proportionnalité entre leur masse et celle du bulbe de la galaxie suggère que les trous noirs et les galaxies évoluent en symbiose : l'énergie énorme libérée 
par les noyaux actifs, lorsque la matière tombe sur le trou noir, produit une rétroaction, capable d'éjecter une grande partie du gaz des galaxies et de modérer ainsi leur formation d'étoiles. Les simulations cosmologiques de formation des galaxies doivent utiliser cette rétroaction pour reproduire les observations. La série de cours fera le point de nos connaissances sur la croissance cosmique des trous noirs, qui suit de près le taux de formation d'étoiles dans l'Univers. Toutefois, de nombreux problèmes demeurent : comment former des trous noirs d'un milliard de masses solaires en moins d'un milliard d'années, pour expliquer l'observation de quasars à $\mathrm{z}=6$ ? La croissance des trous noirs est en effet limitée par la pression de radiation (limite d'Eddington). Nous ferons le point sur le trou noir le mieux connu, celui du centre de la Voie lactée, dont l'horizon va bientôt être dévoilé par Gravity et les interféromètres à longue base (Event Horizon Telescope). Les fusions de trous noirs supermassifs, qui accompagnent chaque fusion de galaxies, pourront très bientôt être détectées par les ondes gravitationnelles et le chronométrage des pulsars.

\section{Cours 1 - Physique des trous noirs, noyaux actifs et quasars}

Les trous noirs sont des objets si compacts que même la lumière ne peut en sortir. Il en existe de deux sortes : les trous noirs de masse stellaires, produits par l'explosion en fin de vie des étoiles massives, et les trous noirs supermassifs, de l'ordre de un million à plusieurs milliards de masses solaires. S'il existe dans chaque galaxie des milliards de trous noirs de la première sorte, il n'existe qu'un seul trou noir supermassif, au centre de chaque galaxie. Les trous noirs actifs sont à l'origine du phénomène de quasars, découverts par Maarten Schmidt en 1964. Leurs particularités remarquables, comme l'existence de jets superluminiques, viennent de l'énergie gravitationnelle colossale libérée par la chute de matière sur le trou noir. Ces trous noirs sont toutefois limités dans leur accrétion de matière par la luminosité d'Eddington, et ils sont obligés de rejeter une grande partie de cette matière, d'où l'existence de vents galactiques et jets. Pour expliquer la grande variété de phénomènes observés, liés aux trous noirs, les astrophysiciens ont bâti le paradigme d'unification: une grande partie de cette variété est due à des phénomènes géométriques et de projection sur la ligne de visée de l'observateur (galaxies de Seyfert 1 ou 2, quasars, ou blazars, etc.). D'autres phénomènes peuvent être expliqués par la physique du disque d'accrétion: soit il existe un disque mince, optiquement épais, au rayonnement de corps noir, dans le cas d'un taxi d'accrétion proche d'Eddington, soit un ADAF (advection-dominated accretion flow), lorsque le rayonnement est inefficace. Le disque d'accrétion commence à s'évaporer, et forme une couronne, à l'origine de rayons X durs.

\section{Cours 2 - Le trou noir au centre de notre Galaxie}

Nous connaissons bien le trou noir supermassif au centre de notre Galaxie, la Voie lactée. C'est de loin le plus proche, sa masse est de quatre millions de masses solaires. Le mouvement propre des étoiles autour a été suivi depuis plus de vingt ans : les étoiles sont animées de vitesse de plus de $1000 \mathrm{~km} / \mathrm{s}$, et ont une trajectoire parfaitement képlériennes. Notre trou noir n'est pas actif, il montre tout de même quelques signes d'émission radio et en rayons $\mathrm{X}$, avec des sursauts quasi périodiques sur des échelles de temps de l'ordre du quart d'heure. Paradoxalement, il existe des étoiles jeunes autour du trou noir, alors que celui-ci devrait empêcher leur condensation, à cause du cisaillement intense des forces de marée. Bien que le trou noir ne soit pas actif aujourd'hui, il l'a été dans un passé proche, ainsi que le montrent 
des rayonnements multi-longueurs d'onde variables sur des dizaines d'années dans le voisinage. Au printemps 2018, l'étoile S2 la plus proche du trou noir est passée au péricentre, et l'instrument GRAVITY (interféromètre proche infrarouge du VLTI de l'ESO) l'a suivie avec une très grande résolution spatiale (plusieurs microarcsecs), afin de tester la théorie de relativité générale en champ fort.

\section{Cours 3 - Coévolution cosmique trous noirs/galaxies}

Il existe une relation étroite entre les masses des trous noirs supermassifs et la masse du bulbe de leurs galaxies hôtes : la masse du trou noir est de l'ordre de $0,2 \%$ de la masse du bulbe. La masse des trous noirs est connue soit par la dynamique directe du Viriel, soit par la mesure de l'émission de masers radio $\left(\mathrm{OH}, \mathrm{H}_{2} \mathrm{O}\right.$, etc.), ou par cartographie de réverbération. Une relation d'échelle a été mise en évidence entre la luminosité du noyau actif et la taille de la région BLR (à raies larges, ou disque d'accrétion). Ainsi, la mesure du spectre (largeur de raies) et de la luminosité du noyau actif, permet aussi d'en déduite la masse, moyennant un facteur d'inclinaison sur la plan du ciel, qui ne peut être estimé que de façon statistique. Des trous noirs obèses ont été mesurés, surtout dans les amas de galaxies. Plusieurs théories sont avancées pour expliquer la proportionnalité entre masses des trous noirs et des bulbes, et notamment les effets de rétroaction des noyaux actifs, qui permettent de former des étoiles au centre, pendant que le trou noir éjecte la matière.

\section{Cours 4 - Fusion de galaxies et trous noirs binaires}

Lors de la fusion de deux galaxies, les trous noirs supermassifs en leurs centres vont se rapprocher, interagir par friction dynamique avec les étoiles du bulbe, et progressivement ralentir pour former une binaire. La présence de gaz accélère la fusion qui, dans le cas purement stellaire, peut prendre un temps assez long, avant de ralentir par émission d'ondes gravitationnelles. La fusion des trous noirs pourrait expliquer en partie la relation d'échelle entre les masses trous noirs-bulbes. Le temps de fusion doit être en général très rapide, car il est assez rare d'observer des binaires de trous noirs au centre des galaxies. Lorsqu'un des noyaux est actif, et émet des jets radio, la présence de binaires pourrait expliquer la précession et la réorientation des jets. Comment se forment les trous noirs au début de l'Univers? Il semblerait que les tous noirs croissent plus vite que leurs bulbes au départ, étant donné la masse des trous noirs au redshift $\mathrm{z}=6$. La détection récente de fusion de trous noirs de masse stellaire par ondes gravitationnelles (LIGO et Virgo) est très encourageante pour la détection future dans l'espace (LISA) de fusions de trous noirs plus massifs, et donc d'ondes gravitationnelles de plus grande longueur d'onde.

\section{RECHERCHE}

Durant cette année académique 2017-2018, notre équipe a travaillé sur un certain nombre de résultats obtenus avec l'interféromètre ALMA, que ce soit des flots moléculaires détectés autour de galaxies à noyaux actifs proches (Alonso-Herrero et $a l$.), des flambées de formation stellaire (Perreira-Santella et al.), ou même des absorptions moléculaires devant les quasars (Wiklind et al.). L'absorption moléculaire devant les quasars lointains est très rare, nous ne connaissons que cinq systèmes dans tout le ciel, en ce qui concerne les distances cosmologiques. Pourtant, cette technique est très complémentaire de l'émission, et permet de sonder les propriétés physiques du milieu interstellaire à toutes les époques. Pour le système 
considéré, il s'agit d'un absorbant à la moitié de l'âge de l'Univers. C'est l'un des trois systèmes où l'absorbant est aussi la galaxie lentille qui amplifie le quasar lointain. Nous avons pu détecter $\mathrm{CO}$ et $\mathrm{HCO}^{+}$dans les deux images, qui sont séparées de $5 \mathrm{kpc}$ dans le plan de la lentille. Les deux raies sont séparées de $215 \mathrm{~km} / \mathrm{s}$, indiquant un système en rotation assez incliné dans le plan du ciel. Le rapport d'abondance $\mathrm{CO} / \mathrm{HCO}^{+}$étant deux à trois fois plus fort qu'attendu, il semblerait que la ligne de visée corresponde à un nuage plus dense que pour les autres systèmes.

Nous avons aussi exploré les galaxies dans les proto-amas, les amas de galaxies les plus lointains identifiés, soit à $\mathrm{z}=1,4$, i.e. quatre milliards d'années après le Big Bang (Casasola et al., Castignani et al.). Pour repérer les amas de galaxies si lointains, on utilise fréquemment la technique de recherche des radiogalaxies ou des noyaux actifs. Avec l'interféromètre NOEMA, nous avons pu détecter du gaz moléculaire dans deux de ces galaxies, qui forment de nombreuses étoiles. Bien que d'énormes quantités de gaz moléculaire aient été trouvées (plus que dix milliards de masses solaires), le temps de consommer ce gaz par la formation actuelle d'étoiles est très court (quelques centaines de millions d'années). Ce temps de déplétion est dix fois inférieur à ce qui se passe pour les galaxies normales dans le champ. Sans doute l'alimentation de gaz est-elle interrompue dans l'amas de galaxies, préfigurant un arrêt proche de la formation d'étoiles.

Notre Galaxie, la Voie lactée, est celle qui présente le plus de détails quant à son évolution et sa dynamique. Récemment, le satellite GAIA a révélé une grande moisson de résultats, concernant les distances et les vitesses d'étoiles, que ce soit dans le disque mince, le disque épais, ou le halo stellaire. Nous avons continué nos simulations numériques de l'influence de la barre, et des disques minces et épais dans la métallicité et les gradients d'abondances (Fragkoudi et al.), de manière à pouvoir comparer les simulations et les observations. Nous avons montré comment l'existence de la barre pouvait diminuer la formation d'étoiles, à cause du cisaillement du gaz sur les orbites $\mathrm{x} 1$, bien que l'accumulation du gaz aux résonances produise une flambée dans les anneaux à l'ILR (Khoperskov et al.). Enfin, nous avons étudié en détail les processus de migration radiale, dus à la présence de la corotation dans une galaxie spirale barrée (Halle et al.). Nous avons pu montrer que les écarts effectués par les étoiles sont surtout dus à l'ellipticité de leurs orbites (blurring), encore plus que le changement de leur centre guide, par échange de moment angulaire (churning). Pour qu'une forte migration radiale ait lieu, il faut que la vitesse de la barre évolue significativement, ce qui se produit couramment dans les galaxies spirales barrées.

\section{PuBLications}

ACERO F. et al.., French SKA White Book. The French Community towards the Square Kilometre Array, 2018 [arXiv:1712.06950].

Alonso-Herrero A., Pereira-Santaella M., García-Burillo S., Davies R.I., Combes F., Asmus D., Bunker A., Díaz-Santos T., Gandhi P., GonzÁlez-Martín O., Hernán-Caballero A., Hicks E., Hönig S., Labiano A., LeVenson N.A., Packham C., Almeida C.R., Ricci C., Rigopoulou D., Rosario D., SANi E. et WARD M.J., « Resolving the nuclear obscuring disk in the Compton-thick Seyfert galaxy NGC5643 with ALMA », The Astrophysical Journal, vol. 859, no 2, 2018, p. 144, DOI : 10.3847/1538-4357/aabe30 [arXiv:1804.04842]. 
Bicalho I.C., Combes F., Rubio M., Verdugo C. et Salome P., «AlMA CO(2-1) observations in the XUV disk of M83», Astronomy and Astrophysics, à paraître [arXiv:1807.01373].

Boselli A. et al., «A virgo environmental survey tracing ionised gas emission (VESTIGE). I : Introduction to the survey », Astronomy and Astrophysics, vol. 614, 2018, A56, DOI : 10.1051/0004-6361/201732407 [arXiv:1802.02829].

Casasola V., Magrini L., Combes F., Sani E., Fritz J., Rodighiero G., Poggianti B., BIANCHI S. et LIUZZO E., «Spectroscopic characterization of the protocluster of galaxies around 7C 1756+6520 at z $~ 1.4$ », Astronomy and Astrophysics, à paraître [arXiv:1806.09493].

Castignani G., Combes F., Salomé P., Andreon S., Pannella M., Heywood I., Trinchieri G., CiCONE C., DAVIES L.J., OWEN F.N. et RAICHOOR A., « Molecular gas in two companion cluster galaxies at $\mathrm{z}=1.2 »$, Astronomy \& Astrophysics, à paraître [arXiv:1806.01826], DOI : 10.1051/0004-6361/201832887.

COMBES F., «AGN feedback and its quenching efficiency », Frontiers in Astronomy and Space Sciences, 2017, vol. 4, no 10, DOI : 10.3389/fspas.2017.00010 [arXiv:1707.09621].

Combes F., «Closing remarks and Outlook », in C. ChiAPPINI, I. MincheV, E. STARKenbug et M. VALENTini (dir.), Redicovering our Galaxy, 2017 [arXiv: 1707.07602].

Combes F., «Molecular gas in distant galaxies from ALMA studies », Astronomy and Astrophysics, à paraître [arXiv: 1806.06712].

Combes F., «Secular evolution of Milky Way-type galaxies », in ReYlé C., MATTEO P.D., Herpin F., LAGADEC E., LANCON A. et ROYER F. (dir.), SF2A Proceedings, 2017, p. 237-240, [arXiv:1707.08733].

COMBES F., « Star formation efficiency », Galaxy Evolution Across Time, vol. 65, 2017, DOI : 10.5281/zenodo.809178.

DE BLOK W.J.G. et al., "An overview of the MHONGOOSE survey: Observing nearby galaxies with MeerKAT », Proceedings of Science, 2017, http://arxiv.org/abs/1709.08458 [arXiv:1709.08458].

Fragkoudi F., Di Matteo P., Haywood M., Khoperskov S., Gómez A., Schultheis M., COMBES F. et SEMELIN B., «What the Milky Way bulge reveals about the initial metallicity gradients in the disc », Astronomy and Astrophysics, vol. 607, 2017, L4, DOI : 10.1051/00046361/201731597 [arXiv:1710.06864].

Fragkoudi F., Di Matteo P., HAYwood M., Schultheis M., KhopersKov S., Gómez A. et COMBES F., «The disc origin of the Milky Way bulge: Dissecting the chemo-morphological relations using N-body simulations and APOGEE », Astronomy \& Astrophysics, à paraître [arXiv:1802.00453], DOI : 10.1051/0004-6361/201732509.

Garcia-Burillo S., Viti S., Combes F., Fuente A., Usero A., Hunt L.K., Martin S., Krips M., Aalto S., Aladro R., Almeida C.R., Alonso-Herrero A., Casasola V., Henkel C., Querejeta M., Neri R., Costagliola F., TACCONi L.J. et VAN DER Werf P.P., « ALMA imaging of $\mathrm{C}_{2} \mathrm{H}$ emission in the disk of NGC 1068 », Astronomy and Astrophysics, vol. 608, 2017, A56, DOI : 10.1051/0004-6361/201731862 [arXiv:1709.05895].

Gomez A., Di Matteo P., Schultheis M., Fragkoudi F., Haywood M. et Combes F., «Searching for a kinematic signature of the moderately metal-poor stars in the Milky Way bulge using N-body simulations », Astronomy and Astrophysics, à paraître [arXiv:1803.09626].

GUPTA N., SRIANAND R., BAAN W. et al., « The MeerKAT Absorption Line Survey (MALS) », MeerKAT Science : On the Pathway to the SKA, 2017 [arXiv:1708.07371].

Halle A., Di Matteo P., Haywood M. et Combes F., « Radial migration in a stellar galactic disc with thick components », Astronomy \& Astrophysics, à paraître, DOI : 10.1051/00046361/201832603 [arXiv:1801.02403]. 
Hamer S.L., Fabian A.C., Russell H.R., Salomé P., Combes F., Olivares V., Polles F.L., EDGE A.C. et BECKMANN R.S., "Discovery of a diffuse optical line emitting halo in the core of the Centaurus cluster of galaxies : Line emission outside the protection of the filaments ", Monthly Notices of the Royal Astronomical Society, à paraître [arXiv:1803.09765].

Henkel C., Muehle S., Bendo G., Jozsa G.I.G., Gong Y., Viti S., Aalto S., Combes F., Garcia-Burillo S., Hunt L.K., Mangum J., Martin S., Muller S., Ott J., Van DER Werf P., Malawi A.A., Ismail H., Alkhuja F., Asiri H.M., Aladro R., Alves F., Ao Y., BaAn W.A., Costagliola F., Fuller G., Greene J., Impellizzeri C.M.V., Kamali F., Klessen R.S., Mauersberger R., Tang X.D., Tristram K., Wang M. et Zhang J.S., « Molecular line emission in NGC 4945, imaged with ALMA », Astronomy and Astrophysics, à paraitre [arXiv:1802.09852].

Khoperskov S., Di Matteo P., HaYwood M. et Combes F., « Stellar metallicity variations across spiral arms in disk galaxies with multiple populations », Astronomy and Astrophysics, vol. 611, 2018, L2, DOI : 10.1051/0004-6361/201732521 [arXiv:1801.08711].

Khoperskov S., Haywood M., Di Matteo P., Lehnert M.D. et Combes F., «Bar quenching in gas-rich galaxies », Astronomy \& Astrophysics, 2018, vol. 609, A60, DOI : 10.1051/0004-6361/201731211 [arXiv: 1709.03604].

Krogager J.-K., Gupta N., Noterdaeme P., Ranjan A., Fynbo J.P.U., SRianand R., PetiTJEAn P., Combes F. et MAHABAL A., «MALS-NOT : Identifying radio-bright quasars for the MeerKAT absorption line survey », The Astrophysical Journal Supplement Series, vol. 235, $\mathrm{n}^{\mathrm{o}}$ 1, 2018, p. 10, DOI : 10.3847/1538-4365/aaab51 [arXiv:1802.00482].

Krumpe M., Husemann B., Tremblay G.R., Urrutia T., Powell M., Davis T.A., SCharwächter J., DeXter J., Busch G., COMbes F., Croom S.M., ECKART A., MCElRoY R.E., PEREZ-Torres M. et LEUNG G., « The Close AGN Reference Survey (CARS): Mrk 1018 halts dimming and experiences strong short-term variability», Astronomy and Astrophysics, vol. 607, 2017, L9, DOI : 10.1051/0004-6361/201731967 [arXiv:1710.09382].

MelChioR A.-L. et COMBES F., «Exhaustion of the gas next to M31's supermassive black hole », Astronomy and Astrophysics, vol. 607, 2017, L7, DOI : 10.1051/0004-6361/201730910 [arXiv: 1710.05710].

Oosterloo T., Oonk J.B.R., Morganti R., Combes F., Dasyra K., Salome P., VLAHAKIS N. et TADHUNTER C., «Properties of the molecular gas in the fast outflow in the Seyfert galaxy IC 5063», Astronomy and Astrophysics, vol. 608, 2017, A38, DOI : 10.1051/0004-6361/201731781 [arXiv:1710.01570].

Pereira-Santaella M., Colina L., Garcia-Burillo S., Combes F., Emonts B., Aalto S., Alonso-Herrero A., Arribas S., Henkel C., Labiano A., Muller S., Lopez J., RigOPOUlou D. et VAN DER WeRF P., "Spatially resolved cold molecular outflows in ULIRGs », Astronomy and Astrophysics, à paraître [arXiv:1805.03667], DOI : 10.1051/00046361/201833089.

Powell M.C., Husemann B., Tremblay G.R., Krumpe M., Urrutia T., Baum S.A., Busch G., Combes F., Croom S.M., Davis T.A., Eckart A., O’Dea C.P., PÉrez-Torres M., SCHARWÄChter J., SMirnova-Pinchukova I. et URRY C.M., «The Close AGN Reference Survey (CARS): No evidence of galaxy-scale hot outflows in two nearby AGN », Astronomy and Astrophysics, à paraître [arXiv:1807.00839].

Russell H.R., Mcnamara B.R., Fabian A.C., Nulsen P.E., Combes F., Edge A.C., Hogan M.T., Mcdonald M., Salomé P., Tremblay G. et Vantyghem A.N., "Close entrainment of massive molecular gas flows by radio bubbles in the central galaxy of Abell 1795 », Monthly Notices of the Royal Astronomical Society, vol. 472, no 4, 2017, p. 4024-4037, DOI : 10.1093/mnras/stx2255 [arXiv:1708.08935].

Salomé Q., Salomé P., Miville-Deschênes M.-A., Combes F. et Hamer S., «Inefficient jet-induced star formation in Centaurus A: High resolution ALMA observations of the 
northern filaments », Astronomy and Astrophysics, vol. 608, 2017, A98, DOI : 10.1051/00046361/201731429 [arXiv:1710.09851].

Tacconi L.J., Genzel R., Saintonge A., Combes F., García-Burillo S., Neri R., Bolatto A., Contini T., Schreiber N.M.F., Lilly S., Lutz D., Wuyts S., Accurso G., Boissier J., Boone F., Bouché N., Bournaud F., Burkert A., CArollo M., CoOper M., Cox P., Feruglio C., Freundlich J., Herrera-Camus R., Juneau S., Lippa M., NaAb T., Renzini A., Salome P., Sternberg A., Tadaki K., Übler H., Walter F., Weiner B. et WEISS A., "PHIBSS : Unified scaling relations of gas depletion time and molecular gas fractions », The Astrophysical Journal, 2018, vol. 853, n 2, p. 179, DOI : 10.3847/1538-4357/ aaa4b4 [arXiv: 1702.01140].

Vantyghem A.N., McNamara B.R., Edge A.C., Combes F., Russell H.R., Fabian A.C., Hogan M.T., MCDONALd M., NULSEN P.E.J. et SALOMÉ P., « A ${ }^{13} \mathrm{CO}$ detection in a brightest cluster galaxy », The Astrophysical Journal, 2017, vol. 848, n 2, p. 101, DOI : 10.3847/15384357/aa8fd0 [arXiv:1709.09679].

WiKLIND T., COMBES F. et KANEKAR N., «ALMA observations of molecular absorption in the gravitational lens PMN 0134-0931», The Astronomical Journal, à paraitre [arXiv:1804.05377], http://arxiv.org/abs/1804.05377. 
